\title{
Major Trends in Device to Device Communications Research: A Bibliometric Analysis
}

\author{
Fadhil Mukhlif ${ }^{1,}{ }^{*}$, Student Member, IEEE, James Oluwaseyi Hodonu-Wusu², Kamarul Ariffin Bin Noordin ${ }^{1}$, \\ Zarinah Mohd Kasirun ${ }^{2}$ \\ ${ }^{1}$ Department of Electrical Engineering, Faculty of Engineering, University of Malaya, Kuala Lumpur, Malaysia \\ ${ }^{2}$ Faculty of Computer Science and Information Technology, University of Malaya, Kuala Lumpur, Malaysia \\ *Corresponding Author: fadhil.researcher@gmail.com
}

\begin{abstract}
The quantitative study of bibliographic material is referred to as bibliometric analysis. It presents a general overview of a research area where the published works can be classified based on countries, authors, publication years, keywords, and fields of research. This study aims to provide a bibliometric analysis of the research carried out in the field of device to device (D2D) communications in the past decade. The primary objective of this study is to find some of the most relevant and influential research papers in this field and define the newest trends as per the information available in the Web of Science database. During the course of this study, numerous classifications have been made based on different parameters such as the most productive and influential authors, and the most influential keywords and journals. The conclusions of this study are in line with the common wisdom, although certain deviations have been found.
\end{abstract}

Keywords-D2D; bibliometric; wireless communication; networking; $5 G$

\section{INTRODUCTION}

The proliferation of cellular communication networks from the first generation $(1 \mathrm{G})$ to the present generation $(5 \mathrm{G})$ is an outcome of the explosive growth in data demand that has been the product of two forces: increasing numbers of subscribers adopting advanced mobile data devices and increasing use of mobile data services by the subscribers that are using these devices. The use of the latest generation of smart devices and USB data modems for computers has created immense growth in the network data traffic. The advancement in cellular communication networks offers better network capacity to meet the growing data and communication needs of subscribers through higher data rate. The data rate is at its highest in the present generation of networks. However, to enable even better device to device (D2D) communication at present as well as in the future, there has to be an augmented system capacity and system throughput [1] that allows the mobile network operators (MNOs) to offer flexibility in usage.
The D2D communication is an adept technology for the next generation networks (NGNs) that is built to meet the trends of wireless communication. The D2D communication technology plays a significant role in enabling today's emerging technologies. The first four generation of cellular communication networks were entirely dependent on the base station (BS), rendering them to be referred to as network centric. But with the induction of D2D communication, which is regarded as an indispensable part of $5 \mathrm{G}$ networks, a direct transmission between devices is expected [2]. As stated by [3], this transmission between devices has the potential to improve reliability of link, boost ethereal efficiency and system capacity, and enhance performance, while mitigating the issues related to delay and inactivity in the network.

There is a considerable scope to reduce the power consumption in the D2D communication networks, as in these networks, the proximate devices communicate directly by forming direct links. There is also the potential to offload traffic congestion efficiently from the core network and distribute content effectively, something which is very difficult to achieve in the traditional cellular communications networks. Moreover, the use cases of D2D communication networks include social networking, ehealth, smart city, location-aware services and smart grids [4] and [5]. [6] addressed a few use cases of D2D communication networks. Hence, it can be concluded that a wide range of applications are offered by D2D communication technology for the NGNs.

Along with the several benefits of the D2D communication networks, there exists a number of challenges or concerns. These challenges are related to its implementation range that cause interference for the cellular network users, and make controlling of D2D users and peer discovery difficult [1]. The objective of this paper is to provide a bibliometric summary of the research published on D2D communications in recent years. This paper aims to find some of the most relevant research in the field of D2D communications, and identify some of the latest trends as per the information available in the web of science database from 2007 to 2017. 


\section{RESEARCH METHOD}

In order to arrive at our model based on prior literature, a broad search was carried out to collect published research studies on D2D communications. The data was collected from the "Web of Science Core Collection" that constitutes of the Social Sciences Citation Index "SSCI", Science Citation Index Expanded "SCI-EXPANDED", Conference Proceedings Citation Index- Science "CPCI-S", Conference Proceedings Citation Index- Social Science \& Humanities "CPCI-SSH", Arts \& Humanities Citation Index "A\&HCI", and newly included Emerging Sources Citation Index "ESCI" that consists of articles of standard and acceptable quality [7] and [8]. The duration of the outcomes of our search is from 2007 to September 2017. Boolean combinations of the keywords "device to device communications" or "Device to Device Communications" or "D2D Communications" or other related terms are used to retrieve the relevant articles. The first search attempt with the keyword "Device to Device Communications" as the title returned 520 articles. The citations source items indexed within the Web of Science Core Collection are reflected in the following report. A cited reference search is performed to add citations to items that are not indexed within the Web of Science Core Collection.

\section{RESULTS}

\section{A. Bibliometric Analysis Based On Keywords}

This report provides analysis on a ten-year records of Device to Device Communications Research downloaded from Web of Science from 2007 through 2017. The analysis identifies the most prolific authors, institutions, journals, and keywords in the dataset based on the number of occurrences and citation counts. A citation network of the provided records is created and used to identify the important papers according to their in-degree, total citation count and PageRank scores [9]. The analysis finds also often-cited references that were not included in the original dataset downloaded from the Web of Science. The analyzed dataset consists of top 25 keywords as shown in Table I, while Fig. 1. displayed the Network Visualization of the Keywords search results of (D2D) Communications Research trends.

TABLE I. Top 25 Keywords Search Results

\begin{tabular}{|c|c|}
\hline Word & Frequency \\
\hline SYSTEMS & 62 \\
\hline CELLULAR NETWORKS & 59 \\
\hline OPTIMIZATION & 54 \\
\hline RESOURCE-ALLOCATION & 49 \\
\hline LTE-ADVANCED NETWORKS & 48 \\
\hline WIRELESS NETWORKS & 48 \\
\hline CELLULAR NETWORKS & 38 \\
\hline TO-DEVICE COMMUNICATIONS & 38 \\
\hline TO-DEVICE COMMUNICATION & 36 \\
\hline DESIGN & 35 \\
\hline CHALLENGES & 33 \\
\hline
\end{tabular}

\begin{tabular}{|c|c|}
\hline 5G & 33 \\
\hline LTE-ADVANCED NETWORKS & 29 \\
\hline NETWORKS & 28 \\
\hline UNDERLAY & 27 \\
\hline UNDERLAYING CELLULAR NETWORKS & 22 \\
\hline RESOURCE-ALLOCATION & 20 \\
\hline NETWORKS & 19 \\
\hline SELECTION & 16 \\
\hline ALLOCATION & 16 \\
\hline CHANNELS & 14 \\
\hline POWER-CONTROL & 13 \\
\hline PERFORMANCE & 12 \\
\hline D2D COMMUNICATIONS & 12 \\
\hline ALGORITHM & 11 \\
\hline
\end{tabular}

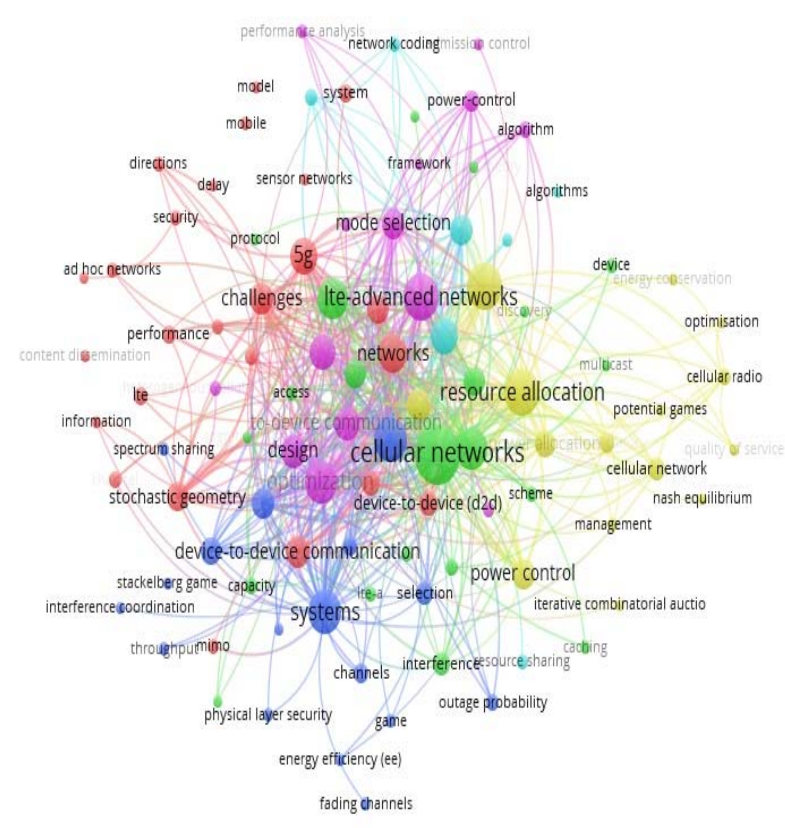

Fig. 1. Network Visualization for Keywords Search Results

\section{B. Analysis Based On Authors}

From this study, the most 10 prolific authors were shown in Table II. These are Li, Y from Southeast University, Nanjing, Peoples Republic of China with a record of (23 articles; 4.42\%), Han, Z from Peking University, Beijing with (16 articles; 3.08\%), Zhang, Y from Nanyang Technology University, Singapore had (12 articles; 2.31\%), Jin, D. P from Tsinghua University, Beijing, Peoples R. China with (11 articles; $2.15 \%)$, while $\mathrm{Li}, \mathrm{G}$. Y from UESTC, National Key Laboratory Communication, Chengdu, Peoples R China., Lei, L from University of Waterloo, Canada and Hossain, E from Peking University, Beijing are with (10 articles each; and 1.92\%) respectively, and lastly Wang, L from Beijing University Posts \& Telecommunication, Beijing; Song, L. Y from Peking 
University, Beijing and Shen, X. M from Arizona State University, USA were with 9 articles each and $1.73 \%$ respectively. This is in agreement with the earlier studies of [7] and [8]. Similar kind of trends have been observed [10] and [11]. Fig. 2. shows the Most Cited Authors. It is evident that some authors are not prolific as others in this area but have higher citation impact such authors include Fodor, Gabor, Feng, Daquan and Lei, Lei and so on.

TABLE II. Top Ten Contributing Authors

\begin{tabular}{|c|c|}
\hline Authors & $\begin{array}{c}\text { Number of Published } \\
\text { Articles }\end{array}$ \\
\hline LI, Y & 23 \\
\hline HAN, Z & 16 \\
\hline ZHANG, Y & 12 \\
\hline JIN, D. P & 11 \\
\hline LI, G. Y & 10 \\
\hline LEI, L & 10 \\
\hline HOSSAIN, E & 10 \\
\hline WANG, L & 9 \\
\hline SONG, L. Y & 9 \\
\hline SHEN, X. M & 9 \\
\hline
\end{tabular}

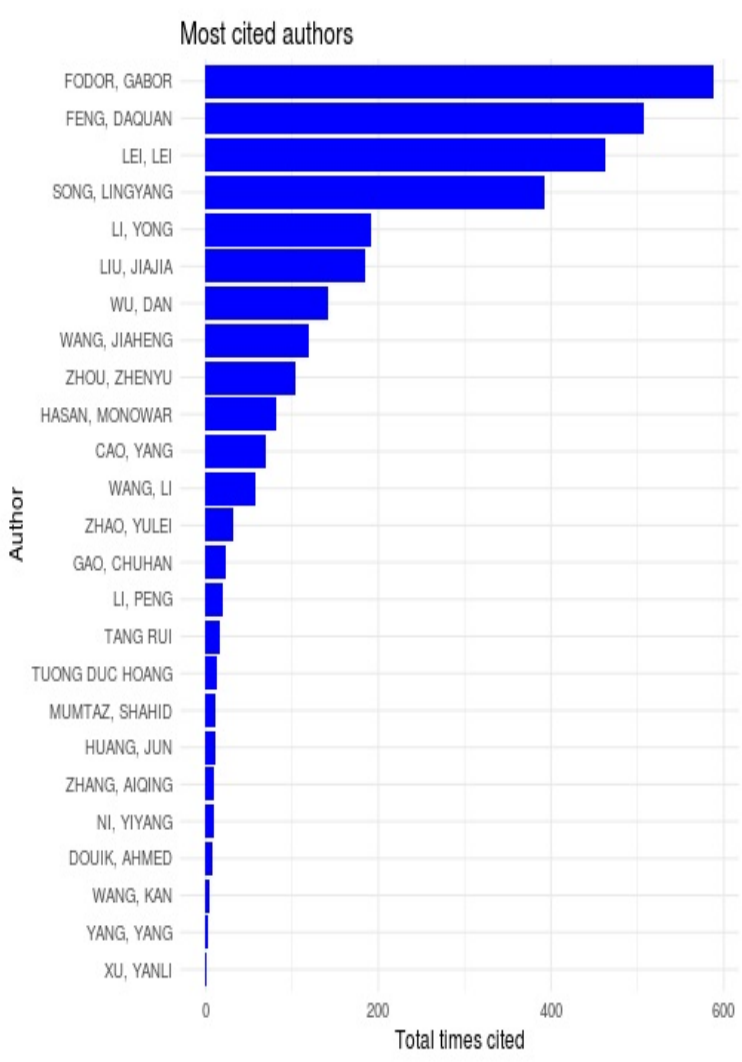

Fig. 2. Most Cited Authors

\section{Analysis Based On Countries}

In presenting the picture of $\mathrm{D} 2 \mathrm{D}$ communication research across different countries/territories, Table III, Fig. 3. and Fig. 4, classifies top 10 countries/territories on the map based on the total number of citations that each country/territory received. Countries like Peoples Republic of China (261, $50.19 \%)$, USA (120, 23.08\%), South Korea $(61,11.73 \%)$, Canada (53, 10.19\%), England (41, 7.89\%), have more than 40 citations in D2D communication research and belong to G5 countries. Of the top 5 countries, only 2 (China and South Korea) belong to Asian region which indicates that they remain the top Asian productive countries in the region. There is no Africa country that made it to the first 10 leading research countries, Others that made it to top ten are Japan with (27, 5.19\%), Finland (21, 4.04\%), Sweden (20, 3.85\%), France $(19,3.65 \%)$, and Taiwan $(18,3.46 \%)$. This means that there is a sharp difference in the study of [7] and [8] as countries like China, improve in their publication citations compared to the earlier studies.

\begin{tabular}{|c|c|c|}
\multicolumn{3}{|c|}{ TABLE III. Top Ten Countries Leading Research } \\
\hline Countries/Territories & Citation Count & $\%$ \\
\hline PEOPLES R CHINA & 261 & 50.19 \\
\hline USA & 120 & 23.08 \\
\hline SOUTH KOREA & 61 & 11.73 \\
\hline CANADA & 53 & 10.19 \\
\hline ENGLAND & 41 & 7.89 \\
\hline JAPAN & 27 & 5.19 \\
\hline FINLAND & 21 & 4.04 \\
\hline SWEDEN & 20 & 3.85 \\
\hline FRANCE & 19 & 3.65 \\
\hline TAIWAN & 18 & 3.46 \\
\hline
\end{tabular}

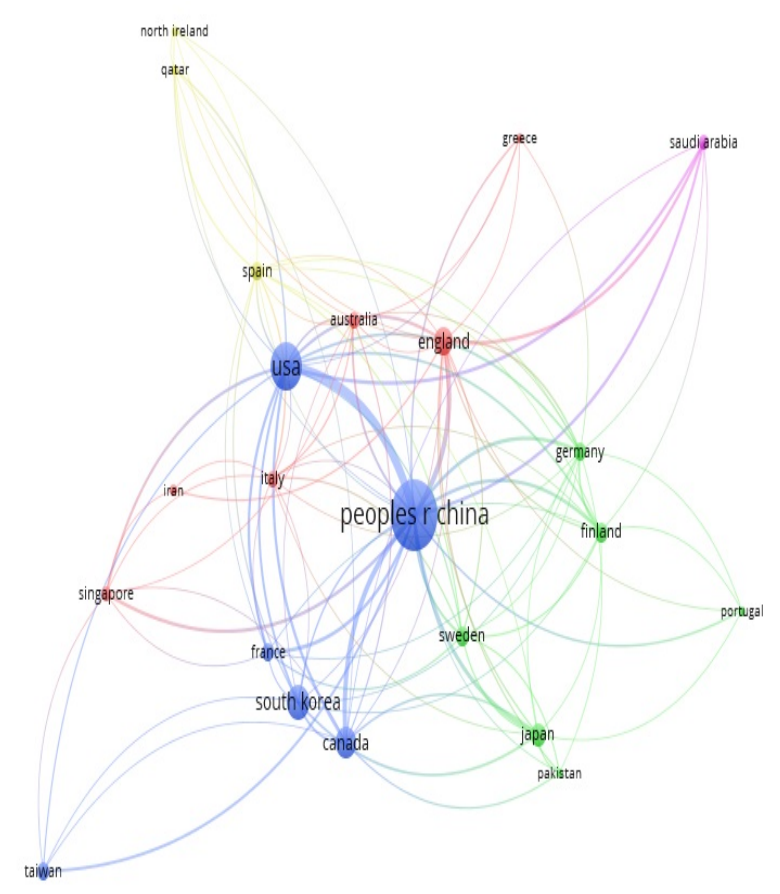

Fig. 3. Network Visualization Map for Top Countries 


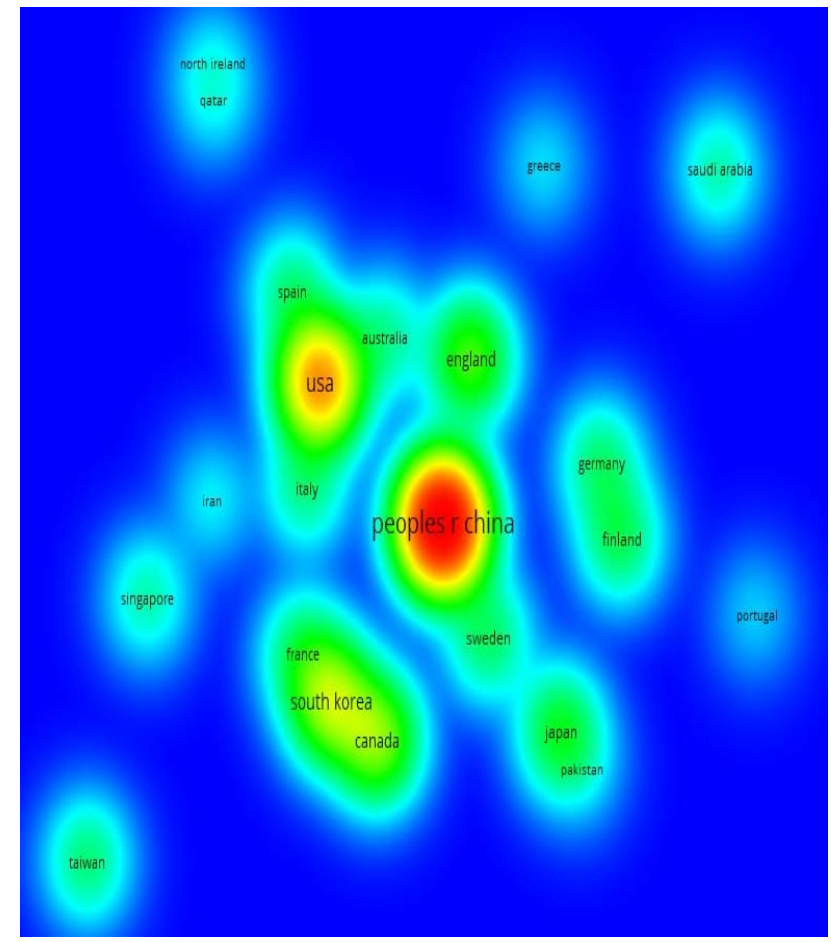

Fig. 4. Density Visualization Map Item for Top Leading Countries

\section{Analysis Based On Publication Types And Year}

There was a total of 520 records analyzed in D2D communication research from 2007 through 2017. However, Table. IV shows document types of the publication with 5 records, out of which the first document type "article" accounted for a $499(95.96 \%)$ documents in D2D communication research. The second document is Editorial Materials with 10 (1.92\%) documents, third and fourth types are reviews and proceedings papers with $8(1.54 \%)$ documents each and lastly correction item with only 2 $(0.39 \%)$ documents were seen. This indicates that many of D2D communication research publications are in form of Journal Articles.

TABLE IV. Number of Publications Based on Document Type
\begin{tabular}{|c|c|c|}
\hline Document Types & $\begin{array}{c}\text { Number of } \\
\text { Documents }\end{array}$ & $\%$ \\
\hline ARTICLE & 499 & 95.96 \\
\hline $\begin{array}{c}\text { EDITORIAL } \\
\text { MATERIAL }\end{array}$ & 10 & 1.92 \\
\hline REVIEW & 8 & 1.54 \\
\hline $\begin{array}{c}\text { PROCEEDINGS } \\
\text { PAPER }\end{array}$ & 8 & 1.54 \\
\hline CORRECTION & 2 & 0.39 \\
\hline
\end{tabular}

In this study as shown in Table $\mathrm{V}$ and Fig. 5, we analyzed the time trend of $\mathrm{D} 2 \mathrm{D}$ communications total publications per year followed by the sum of times D2D communications research publications are cited per year. It is evident that there has been a low increase in the number of citations from 2007 to 2009 and increasing steadily from 2012 to 2017. The lowest number of publications was in the earlier years of the study 2007-2011 with 13(2.52\%) publications while the highest number was in 2017 with 154 (29.62\%) publications (see Table V).
TABLE V. Publication by Year

\begin{tabular}{|c|c|c|}
\hline $\begin{array}{c}\text { Publication } \\
\text { Years }\end{array}$ & $\begin{array}{c}\text { Number of } \\
\text { Publications }\end{array}$ & \% \\
\hline 2017 & 154 & 29.62 \\
\hline 2016 & 151 & 29.04 \\
\hline 2015 & 109 & 20.96 \\
\hline 2014 & 63 & 12.12 \\
\hline 2013 & 20 & 3.85 \\
\hline 2012 & 10 & 1.92 \\
\hline 2009 & 4 & 0.77 \\
\hline 2011 & 3 & 0.58 \\
\hline 2010 & 2 & 0.39 \\
\hline 2008 & 2 & 0.39 \\
\hline 2007 & 2 & 0.39 \\
\hline
\end{tabular}

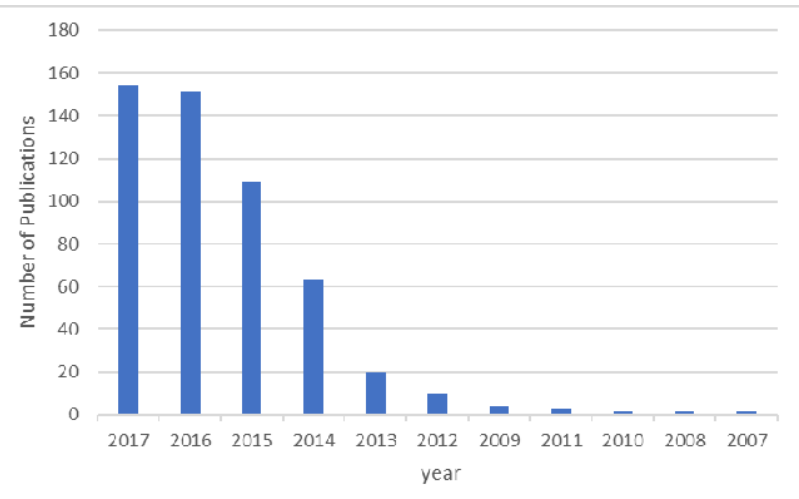

Fig. 5. Distributed of Articles Published per year

\section{E. Bibliometric Analysis Based On Research Area}

Analysis by research areas was conducted using WoS Database for D2D Communications research. A total of 520 records were returned. Telecommunication top the research areas of the authors in D2D Communications research with $423(81.35 \%)$, others in the top five are Engineering 349 (67.12\%), Computer Science 172 (33.08\%), Transportation research $48(8.65 \%)$ and Chemistry $11(2.12 \%)$. The last five of the top 10 research areas in D2D Communications are Instrument Instrumentation 6 (1.15\%), Science Technology and other topics; Physics; and Optics are 5(0.96\%) respectively, the last on the top 10 is Health Care Science Services with 4(0.77\%), as shown in Fig. 6.

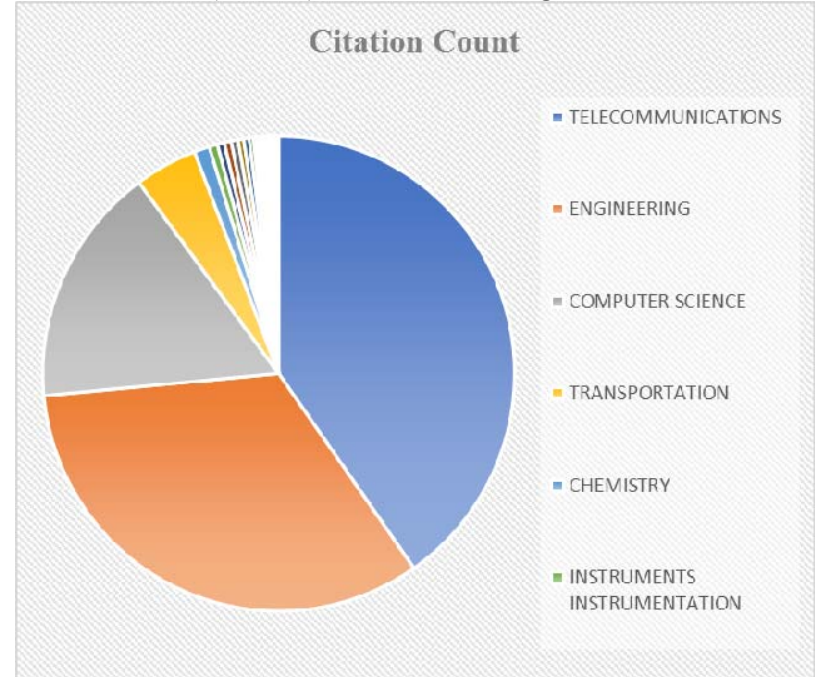

Fig. 6. Main Area of Research in Terms of D2D Communications 


\section{F. Bibliometric Analysis Based On Journals}

There was a total of 520 records analyzed in D2D Communication research from 2007 through 2017. Nevertheless, Table VI shows source of publishing journals with 4 and above citations, out of which the first 10 publishing journals accounted for a $59(11.35 \%)$ that published in D2D Communications research. The top ten journals are IEEE Transactions on Wireless Communications with $59(11.35 \%)$, IEEE transactions on vehicular technology 44(8.467\%), IEEE access research $27(5.19 \%)$, IEEE Journal on selected areas in Communications; and IEEE Communications Letters had 24(4.62\%), IEEE Wireless Communications 23(4.42\%), and the least five of the ten journals are IEEE Communications Surveys and Tutorials and Eurasip Journal on Wireless Communications and Networking are with $6(1.15 \%)$ respectively, Wireless Communications Mobile Computing 5(0.96\%), while Sensors and Mobile Networks Applications Journals have $4(0.77 \%)$ respectively. This indicates that many of D2D Communication research journals are from the IEEE transactions on wireless communications, IEEE transactions on vehicular technology, IEEE access research, IEEE Journal on selected areas in Communications; and IEEE Communications Letters and IEEE Wireless Communications. Fig. 7. shows the most cited publications per journal items in these years of research. From the figure, IEEE Communications Magazine top the most cited publications, followed by the IEEE transactions on wireless communications, IEEE wireless communications, IEEE transactions on communications and IEEE Journal on selected areas in Communications.

TABLE VI. Top 10 Journals for D2D Communication Area

\begin{tabular}{|l|c|c|}
\hline \multicolumn{1}{|c|}{ Journals } & \multicolumn{1}{|c|}{$\begin{array}{c}\text { No. of } \\
\text { Articles }\end{array}$} & \% \\
\hline $\begin{array}{l}\text { IEEE Transactions on Wireless } \\
\text { Communications }\end{array}$ & 59 & 11.35 \\
\hline $\begin{array}{l}\text { IEEE Transactions on } \\
\text { Vehicular Technology }\end{array}$ & 44 & 8.46 \\
\hline IEEE Access & 27 & 5.19 \\
\hline $\begin{array}{l}\text { IEEE Journal on Selected Areas } \\
\text { in Communications }\end{array}$ & 24 & 4.62 \\
\hline IEEE Communications Letters & 24 & 4.62 \\
\hline $\begin{array}{l}\text { IEEE Wireless } \\
\text { Communications }\end{array}$ & 23 & 4.42 \\
\hline $\begin{array}{l}\text { Wireless Personal } \\
\text { Communications }\end{array}$ & 21 & 4.04 \\
\hline IET Communications & 21 & 4.04 \\
\hline $\begin{array}{l}\text { IEEE Communications } \\
\text { Magazine }\end{array}$ & 20 & 3.85 \\
\hline $\begin{array}{l}\text { IEEE Transactions on } \\
\text { Communications }\end{array}$ & 19 & 3.65 \\
\hline
\end{tabular}

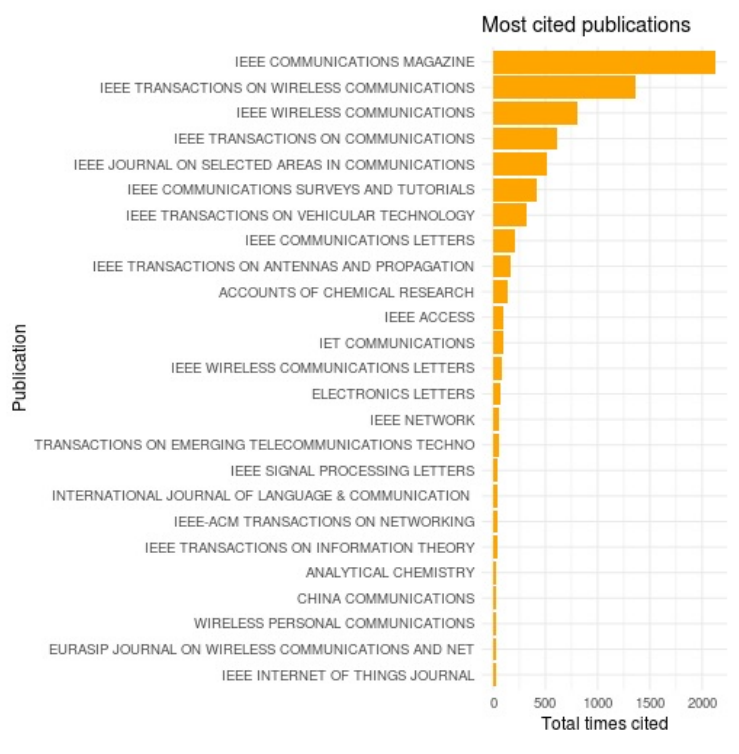

Fig. 7. Most Cited Publications per Journal Items

\section{DISCUSION}

In this study, a bibliometric analysis of all the literature published so far on D2D Communication was conducted. This study was prompted by two factors: first, in spite of the exponential growth in D2D communication, the literature surrounding D2D is still not developed; and second, D2D communication has gained significant attention from the academics as well as the industry. Most of the literature available on D2D communication is based on a technological perspective. The literature or research on the implementation and adoption of D2D communication in business, including areas such as smart cities, smart cars, vehicle to vehicle communications (V2V), effective supply chains, and new age hospitals, is still at a very nascent stage [4] and [5].

The present study offers a bibliometric analysis of the literature already published on D2D communication. Though our study is conducted across a span of 10 years, most of the papers have been published only in the last four years. As suggested by our findings, it is worthwhile to note that the most influential studies have come from a few researchers. The contribution of this study lies in finding the top contributing researchers in the field and their publication years, and in identifying the key research areas, influential keywords, and the top contributing countries. We believe that our analysis plays a vital role in enabling researchers not only to accept the diversity of research in the field, but also help them identify the areas that require additional research. The focus of research need not be only on finding the present and evolving technology solutions for D2D communication [6] and [1], rather it should be to attend to the varied socio-organisational scenarios, both international and local, in which D2D can be embedded [3]. So far, no research has been conducted on the adoption process. There is a considerable scope to conduct studies on the drivers, enablers, barriers and models of D2D adoption by supply chains and organisations, from a socio-organisational as well as a technological perspective. Moreover, there are very limited studies that analyse the relationship between D2D adoption and enhancement of supply chain and 
organisational performance. These kinds of studies are very important and necessary due to the emphasis on sustainability and efficiency in recent times within the supply chain, and to achieve cost-efficiency, low environmental footprint, low energy consumption, high visibility and high transparency in the present systems through the use of technological solutions [3].

This study provides certain additional managerial implications, such as, it facilitates practitioners to recognise the vision and varied applications of D2D, and the scope of research in different clusters. The study recommends that managerial focus should not be only on the selection of technologies, but also on the greater socio-organisational consequences of the D2D adoption for supply chains and organisations. It further highlights the need to attain a holistic understanding of the implications of D2D for managers and decision-makers, so that they become capable of making better decisions with regards to D2D adoption and analysing the required resources necessary for facilitating the transition to the D2D setup and optimising performance.

The primary objective of this study is to find highly influential works related to D2D that give directions to the D2D research community for future research, and also provide implications for decision-makers and managers. The results of this study on D2D research indicate the growing significance of D2D, but at the same time, the study reveals the absence of literature that acknowledges the applications of D2D for supply chains and organisations and considers the wider socio-organisational context. Therefore, most of the highly cited works in the field are dominated and lead by conceptualisations. There are very limited applications of D2D which include case studies that would provide thorough understanding of how D2D emerges, how it is adopted, and what the benefits and challenges of using it. This study highlights the necessity for alternate theories and lenses that must be used to study D2D related phenomena thoroughly. The outcomes of this study, most likely, will be beneficial for scholars in understanding the concept of D2D and the changing research trends in the field of D2D, and in identifying the articles that have been influential in shaping the research and intellectual structure of the D2D field in the past decade.

This study comes with a number of limitations. To begin with, the analysis was carried out on the data derived only from Web of Science (WoS). Next, there might have been a considerable amount of missing or erroneous data in the database that was not identified or rectified. In addition to this, the use of specific keywords in the search for titles might have generated different search results, where the architectural and technological elements of D2D were not taken into consideration. In spite of these shortcomings, this study provides a lot of value and can be used as a reference for research evaluation by grant panels, hiring managers, and reviewers.

\section{CONCLUSION}

This paper is a first attempt to carry out a bibliometric study of D2D communications. 520 articles were collected for the study and it was found that the number of published articles across a range of disciplines has steadily increased over time, implying the growing significance of the field. Most of the influential journals were by researchers from the Peoples Republic of China. Hence, it is concluded that China is leading the scientific community in this particular field. However, it is expected that researchers and scholars from other rapidly growing regions will have considerable influence in the research landscape of this field in the future. Lastly, D2D communication plays a significant role in the next generation of telecommunications, especially with regards to wireless communication and internet of things (IoT) and it will be of extreme relevance in the years to come.

\section{ACKNOWLEDGMENT}

The authors would like to thank the anonymous reviewers for their valuable suggestions and comments on the manuscript.

\section{REFERENCES}

[1] Gandotra, P, and Kumar Jha, R (2016) Device-to-Device Communication in Cellular Networks: A Survey.

[2] Astely,D.,etal., (2013). LTE release 12 and beyond. IEEE Commun. Mag. 51(7),154-160.

[3] Chai, Yingqi, Qinghe, Du, Pinyi, Ren, (2013). Partial time-frequency resource allocation for device-to-device communications underlaying cellular networks. In: Proceedings of 2013 IEEE International Conference on Communications (ICC). IEEE.

[4] Pratas, N.K., Popovski, P., (2013). Low-rate machine-type communication via wireless de- vice-to-device (D2D) links. arXiv preprint arXiv:1305.6783.

[5] Fey, Simon, et al., 2012. Device-to-device communication for Smart Grid devices. In: Proceedings of 2012 3rd IEEE PES International Conference and Exhibition on In- novative Smart Grid Technologies (ISGT Europe). IEEE.

[6] Lei, L., etal., (2012). Operator controlled device-to-device communications in LTE -advanced networks. IEEE Wirel. Commun.19(3),96-104.

[7] Chuang, K.-Y., \& Ho, Y.-S. (2014). A bibliometric analysis on top cited articles in pain research. Pain Medicine, 15(5), 732-744.

[8] Zhu, C., Jiang, T., Cao, H., Sun, W., Chen, Z., \& Liu, J. (2015) Longitudinal analysis of meta-analysis literatures in the database of ISI Web of Science. International Journal of Clinical and Experimental Medicine, 8(3), 3559-3565.

[9] Knutas, A., Hajikhani, A., Salminen, J., Ikonen, J., Porras, J., 2015. Cloud-Based Bibliometric Analysis Service for Systematic Mapping Studies. CompSysTech 2015.

[10] Thompson, D. F., \& Walker, C. K. (2015). A descriptive and historical review of bibliometrics with applications to medical sciences. Pharmacotherapy, 35(6), 551-559. doi:10.1002/phar.1586.

[11] Vali, L., Izadi, A., Jahani, Y., \& Okhovati, M. (2016). Investigating Knowledge Management Status among Faculty Members of Kerman University of Medical Sciences based on the Nonaka Model in 2015.Electronic Physician, 8(8), 2738-2746.doi:10.19082/2738. 